\title{
Gene expression proffiling of the SCN in young and old rhesus macaques
}

\author{
Dominique H Eghlidi', Selva L Luna², Donald I Brown³, Vasilios T Garyfallou4, Steven G Kohama ${ }^{4}$ and \\ Henryk F Urbanski4,5,6
}

1Department of Neurology and Division of Sleep Medicine, Harvard Medical School, Beth Israel Deaconess Medical Center, Boston, Massachusetts, USA 2Escuela de Química y Farmacia, Facultad de Farmacia, Universidad de Valparaíso, Valparaíso, Chile ${ }^{3}$ Instituto de Biología, Facultad de Ciencias, Universidad de Valparaíso, Valparaíso, Chile ${ }^{4}$ Division of Neuroscience, Oregon National Primate Research Center, Beaverton, Oregon, USA ${ }^{5}$ Department of Behavioral Neuroscience, Oregon Health \& Science University, Portland, Oregon, USA ${ }^{6}$ Department of Physiology \& Pharmacology, Oregon Health \& Science University, Portland, Oregon, USA

Correspondence should be addressed to H F Urbanski: urbanski@ohsu.edu

\begin{abstract}
In mammals, the suprachiasmatic nucleus (SCN) is the location of a master circadian pacemaker. It receives photic signals from the environment via the retinal hypothalamic tract, which play a key role in synchronizing the body's endogenously generated circadian rhythms with the 24-h rhythm of the environment. Therefore, it is plausible that age-related changes within the SCN contribute to the etiology of perturbed activityrest cycles that become prevalent in humans during aging. To test this hypothesis, we used gene arrays and quantitative RT-PCR to profile age-related gene expression changes within the SCN of male rhesus macaques - a pragmatic translational animal model of human aging, which similarly displays an age-related attenuation of daytime activity levels. As expected, the SCN showed high expression of arginine vasopressin, vasoactive intestinal polypeptide, calbindin and nuclear receptor subfamily 1, group D, member 1 (NR1D1) (also known as reverse strand of ERBA (REV-ERB $\alpha$ ), both at the mRNA and protein level. However, no obvious difference was detected between the SCNs of young (7-12 years) and old animals (21-26 years), in terms of the expression of core clock genes or genes associated with SCN signaling and neurotransmission. These data demonstrate the resilience of the primate SCN to normal aging, at least at the transcriptional level and, at least in males, suggest that age-related disruption of activity-rest cycles in humans may instead stem from changes within other components of the circadian system, such as desynchronization of subordinate oscillators in other parts of the body.
\end{abstract}

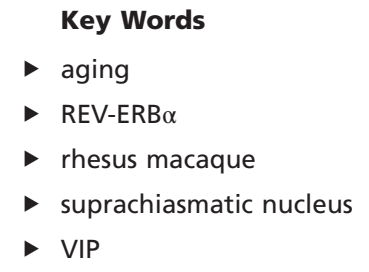

Journal of Molecular Endocrinology (2018) 61, 57-67

\section{Introduction}

The suprachiasmatic nucleus (SCN) of the hypothalamus comprises a heterogeneous population of neuropeptidergic neurons, which play a key role in the circadian organization of many physiological and behavioral rhythms (Hastings et al. 2003, Maywood et al. 2007, Urbanski 2011, Saper
2013, Bailey \& Silver 2014). The core clock mechanism of the SCN is synchronized with the environment via inputs from the retinohypothalamic tract, as well as intra-SCN signaling involving vasoactive intestinal polypeptide (VIP) in the ventrolateral core and arginine vasopressin (AVP) 
in the dorsal part of the SCN shell. Gamma-aminobutyric acid (GABA), which is expressed in all SCN neurons, and diffusible factors such as prokineticin 2 (PK2) in the SCN shell and calbindin (CALB) in a sub-region of the SCN, also play a role in maintaining the integrity of circadian clock and in relaying circadian information to the rest of the body (Gao et al. 1995, Abrahamson \& Moore 2001, Ziegler et al. 2002, Hamada et al. 2003, Aton et al. 2006, Maywood et al. 2006, Cayetanot et al. 2007, Irwin \& Allen 2009, An et al. 2012, 2013, Burton et al. 2016). Although the total number of neurons in the SCN does not change significantly with age (Sartin \& Lamperti 1985, Roberts et al. 2012, Engelberth et al. 2014), there is evidence for a decline in the daily multiunit neural activity rhythm of the SCN (Nakamura et al. 2011), and for some of the SCN neuropetides to show an age-related decrease in expression (Hofman \& Swaab 1994, Hofman et al. 1996, Harper et al. 2008). Additionally, there have been reports of a temporal shift and decrease in VIP and AVP expression in the SCN of rodents and the mouse lemur, a short-lived nocturnal primate species (Roozendaal et al. 1987, Chee et al. 1988, Kawakami et al. 1997, Cayetanot et al. 2005, 2007, Aujard et al. 2006). Furthermore, there is evidence to suggest that the core clock rhythm and internal synchrony of the SCN may change during aging (Weinert 2000, Weinert et al. 2001, Kolker et al. 2003, Nakamura et al. 2011, Farajnia et al. 2012, Bailey \& Silver 2014). Taken together, there is much evidence to suggest that the SCN of humans undergoes significant molecular or physiological changes during aging, which could contribute to the development of age-associated disorders in the elderly, such as perturbed activity-rest cycles. On the other hand, comprehensive gene expression profiling of the human SCN has been impossible to perform, given the difficulty in obtaining human brain tissue without a significant postmortem interval and variable degradation of mRNA. Therefore, in the present study, we used the rhesus macaque as a pragmatic translational animal model for human aging. Our goal was to characterize the neuronal organization of the primate SCN and to determine whether significant age-related changes occur at the gene expression level.

\section{Materials and methods}

\section{Animals}

The study was performed on postmortem tissue obtained from 35 rhesus macaques (Macaca mulatta), which had been cared for by the Division of Comparative Medicine at the Oregon National Primate Research Center in accordance with the National Research Council's Guide for the Care and Use of Laboratory Animals, and initially used in unrelated studies that were approved by the Institutional Animal Care and Use Committee. The animals were housed indoors under controlled environmental conditions: $24^{\circ} \mathrm{C}$ temperature; 12 -hour light, 12-hour darkness photoperiods with lights on at $700 \mathrm{~h}$; regular meals at $\sim 800 \mathrm{~h}$ and $\sim 1500 \mathrm{~h}$ (zeitgeber time (ZT)1 and ZT8, respectively). The animals were fed a High-Protein Monkey Chow (LabDiet, Inc., St. Louis, MO, USA) supplemented with fruit, vegetables and candy; fresh drinking water was available ad libitum. Postmortem tissues from these animals subsequently became available for the present study, which involved immunohistochemical characterization of the SCN as well as gene expression profiling using RNA-sequencing (RNAseq), Affymetrix Rhesus Gene Chip 1.0 ST microarrays and TaqMan quantitative RT-PCR (qRT-PCR).

\section{Characterization of the rhesus macaque SCN}

Because of the paucity of information available about the exact size and location of the SCN in the rhesus macaque brain, especially during aging, we performed a preliminary study in which we used immunohistochemistry to characterize the precise anatomical location of the master circadian clock in both young and old monkeys. A total of 14 SCNs were obtained from young (9-12 years old, $n=3$ ) and old ( 26 years old, $n=11)$ monkeys, all of which were collected at ZT3. The examination focused on the distribution of cells that were immunopositive for AVP, VIP, CALB and REV-ERB $\alpha$ - i.e., neuropeptides known to be associated with the SCN core clock mechanism, as well as its major inputs and outputs.

\section{Quantification of age-related gene expression changes in the SCN}

Taken together, the results from preliminary study enabled us to more-precisely identify and dissect the SCN in frozen hypothalamic blocks from adult male rhesus macaques, and thereby to more accurately quantify agerelated gene expression changes that occur within the SCN. Using gene arrays and qRT-PCR, we compared gene expression profiles between young (7-12 years, $n=6)$ and old (21-26 years, $n=6$ ) males. Postmortem tissues from the young and old animals were all collected during the daytime (1000-1100 h, ZT3-4) and were further processed concomitantly. 


\section{Immunohistochemistry}

Each brain was flushed with $1 \mathrm{~L}$ of $0.9 \%$ saline (at room temperature) and dissected hypothalamic blocks were immersion fixed in a $4 \%$ paraformaldehyde phosphate buffer ( $\mathrm{pH}$ 7.4) for 1 week at $4^{\circ} \mathrm{C}$. Next, the blocks were transferred to a $10 \%$ glycerol in $0.1 \mathrm{M}$ PBS (pH 7.4) containing $2 \%$ DMSO for $24 \mathrm{~h}$, followed by immersion in PBS/DMSO solution containing 20\% glycerol for $72 \mathrm{~h}$. The blocks were then frozen in a beaker of isopentane that was cooled with dry ice and $100 \%$ ethanol and stored at $-80^{\circ} \mathrm{C}$. They were subsequently mounted on a frozen-stage sliding microtome using KPBS and sectioned at $25 \mu \mathrm{m}$; the sections were immediately transferred to a cryoprotectant solution and stored at $-20^{\circ} \mathrm{C}$ until use. Antigen retrieval, followed by single-label immunohistochemistry for AVP, VIP, CALB and REV$\mathrm{ERB} \alpha$, was performed as follows: Floating tissue sections were removed from the cryoprotectant and washed in $0.05 \mathrm{M}$ Tris buffer ( $\mathrm{pH}$ 7.6). Next, the sections were transferred to individual preheated $2 \mathrm{~mL}$ centrifuge tubes filled with $50 \mathrm{mM}$ sodium citrate buffer ( $\mathrm{pH}$ 9.0) and then the tubes were submerged in a hot water bath for $30 \mathrm{~min}$ at $80^{\circ} \mathrm{C}$ (Jiao et al. 1999). After cooling, the sections were rinsed in Tris buffer and incubated in blocking buffer (Tris buffer $+3 \%$ normal donkey serum) for $1 \mathrm{~h}$, to reduce background staining, and then incubated overnight at room temperature in one of the following antibody solutions: rabbit polyclonal anti-AVP (1:2000; LifeSpan Biosciences, Seattle, WA, USA), rabbit polyclonal anti-VIP (1:750; LifeSpan Biosciences), mouse monoclonal antiCALB-D-28K (1:500, Millipore-Sigma, St. Louis, MO, USA) or mouse monoclonal anti-REV-ERB $\alpha$ (1:200; Lifespan Biosciences). After washing in Tris buffer, the sections were incubated for $1 \mathrm{~h}$ in either fluorescein isothiocyanate (FITC)-labeled or tetramethylrhodamine isothiocyanate (TRITC)-labeled secondary antibody (1:200; Jackson ImmunoResearch Laboratories, Inc.) and again washed in Tris buffer. To confirm antibody specificity, the protocol was repeated, except with the primary antibodies preincubated with their corresponding peptides (i.e., AVP (Lifespan Biosciences), VIP (Abcam), CALB (Abcam) and REV-ERB $\alpha$ (LifeSpan Biosciences)) at a concentration of $100-300 \mu \mathrm{g} / \mathrm{mL}$ for $1 \mathrm{~h}$, to which $\leq 300 \mu \mathrm{g}$ was sufficient to eliminate specific labeling in the SCN. Sections were mounted onto poly-L-lysine-coated glass microscope slides and then processed with Autofluorescence Eliminator Reagent (Chemicon). Briefly, the mounted sections were immersed in Tris buffer for $5 \mathrm{~min}, 70 \%$ ethanol for $5 \mathrm{~min}$, Autofluorescence Eliminator Reagent for $7 \mathrm{~min}$, washed in $70 \%$ ethanol and then cover slipped using ProLong
Diamond Antifade Mountant (Thermo Fisher Scientific Inc.). Immunofluorescence microscopy was subsequently performed using a Leica SP5 confocal microscope with Acousto-Optical Beam Splitter. The neuronal distribution pattern of AVP, VIP, CALB and REV-ERB $\alpha$ was examined in 5-7 hemi-lateral coronal SCN sections from each animal.

\section{RNA-seq}

To corroborate the immunohistochemical results, RNAseq was performed on postmortem tissues obtained from three additional adult rhesus macaques (12-13 years). The SCN samples were sub-dissected from brains that had been flushed with $1 \mathrm{~L}$ of $0.9 \%$ saline (at room temperature) and preserved in RNAlater (Ambion) for 1-2 weeks. Based on the immunohistochemical images (Fig. 1), the SCN was localized to the rostral part of the hypothalamus, ventral to the paraventricular nucleus, lateral to the third ventricle and just dorsal of the chiasma. For control purposes, RNA-seq was also performed on the amygdala and adrenal gland, which were collected as previously described (Lemos et al. 2006, Haley et al. 2012). The RNA was isolated using an RNeasy Plus Universal Mini Kit (Qiagen) with two QIAcube Automation Systems (Qiagen). Next, an Agilent 2100 Bioanalyzer (Agilent Technologies) was used to assess the quality of the RNA, and a NanoDrop 2000 (Thermo Fisher Scientific Inc.) was then used to determine the concentration. The RNA was subsequently processed by the Oregon Health and Sciences University (OHSU) Massively Parallel Sequencing Shared Resource. Library preparation and subsequent deep sequencing of rhesus macaque RNA was completed by the Integrated Genomics Laboratory at OHSU. Singleend read sequencing of samples was performed on lanes of an Illumina HiSeq 2000 flow cell. Reads were evaluated for elevated duplication levels (indicating possible adapter contamination or PCR bias) and sequence quality using FastQC (v0.10.1) software (Babraham Bioinformatics). Adaptive quality trimming was performed using a Trimmomatic (v0.30) algorithm favoring sequence quality over read length (Lohse et al. 2012). All other settings were left at default for single-end read data. The remaining reads were aligned with STAR (v.2.3.0), a commonly used RNA-seq aligner, using the most recent rhesus macaque genome build and gene annotation (BioProject accession: PRJNA214746). Alignment parameters were similar to the default parameters, with the exception of allowing three mismatches per 100-bp. HTSeq (v0.5.4p3) was used to count unique reads from the STAR alignment (Anders \& Huber 2010). Count data were based on exon boundaries 

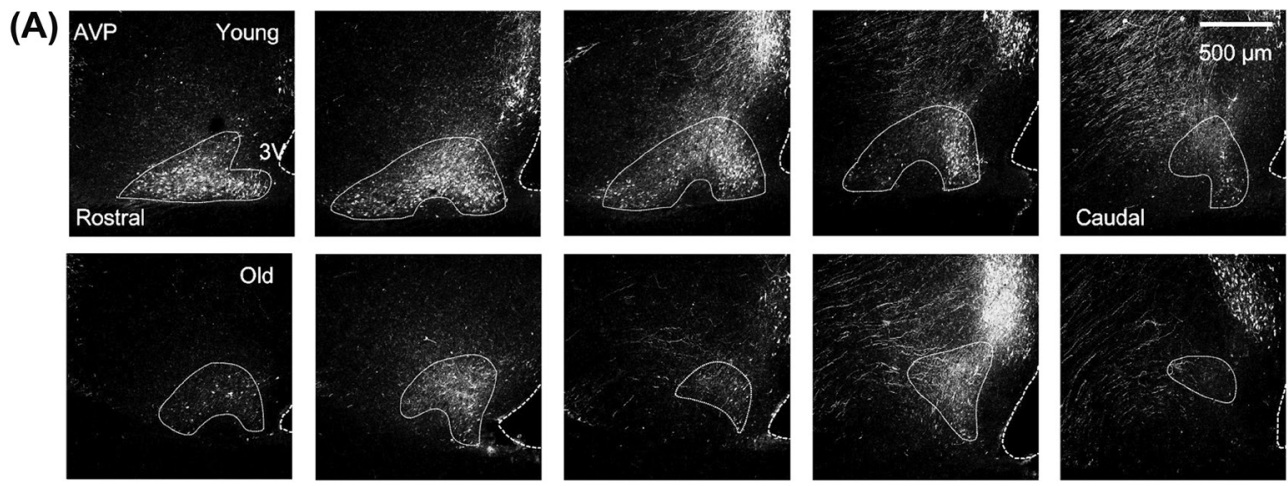

(B)
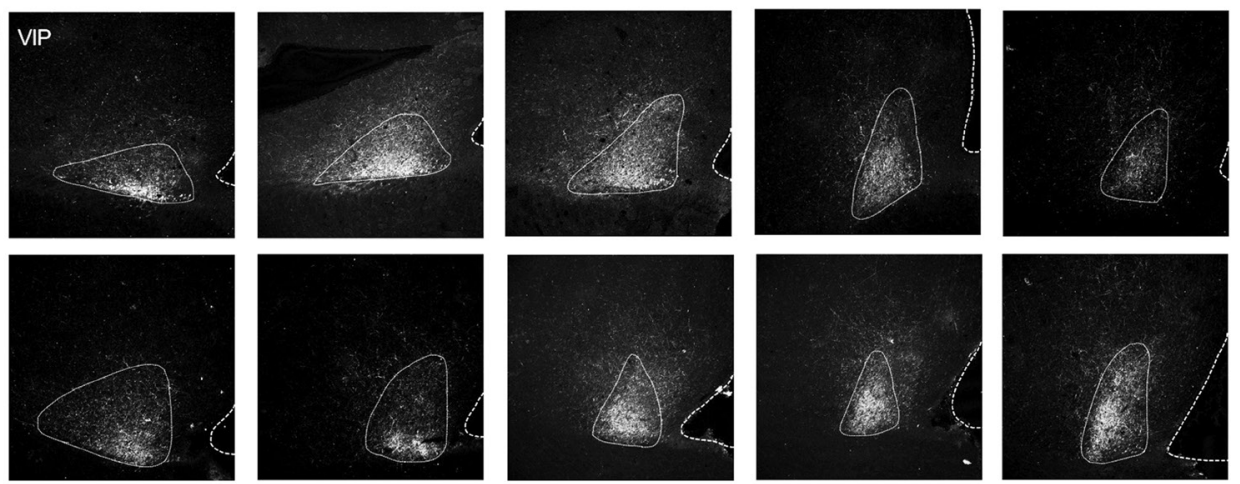

(C)
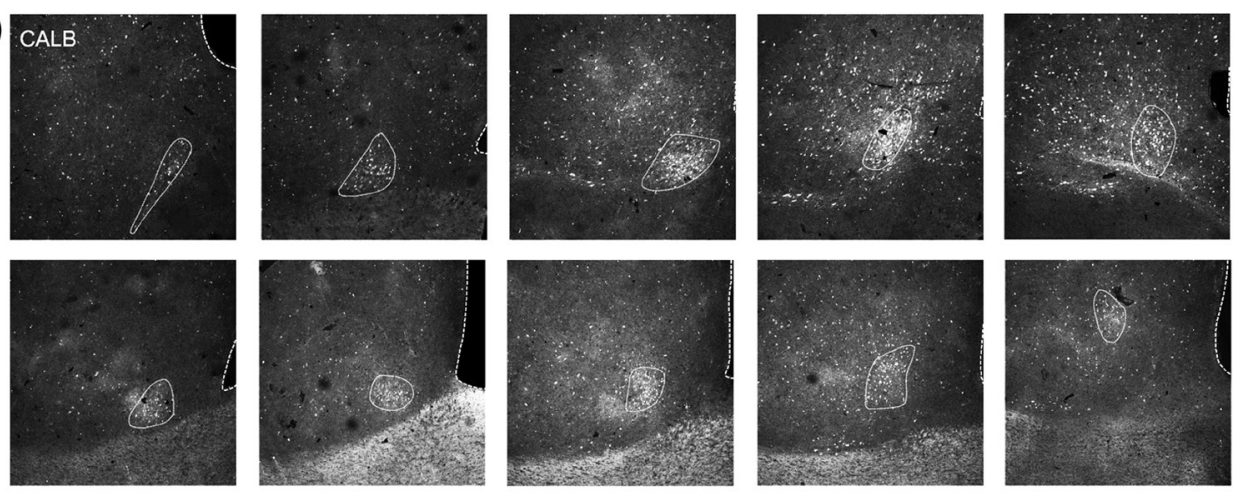

(D)
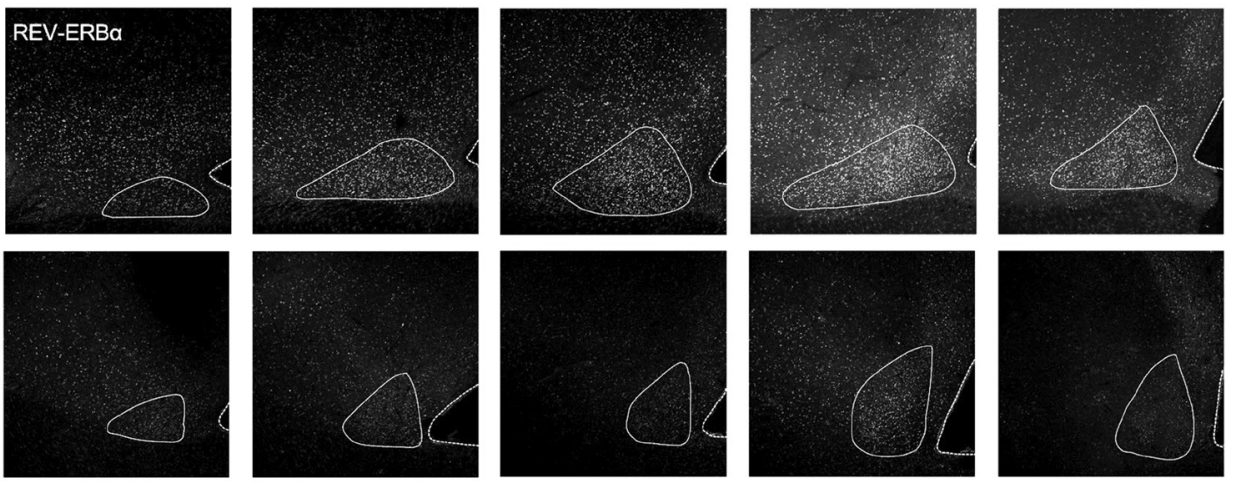

Figure 1

Rostral-to-caudal organization of the rhesus macaque suprachiasmatic nucleus revealed using immunohistochemistry. Unilateral coronal sections from representative young and old animals are depicted in the upper and lower panels of each pair, respectively. (A) Arginine vasopressin (AVP).

(B) Vasoactive intestinal peptide (VIP). (C) Vitamin D-dependent calcium-binding protein (CALB). (D) Reverse strand of ERBA (REV-ERB $\alpha$ ), also known as nuclear receptor subfamily 1 , group D, member 1 (NR1D1). The dotted white lines on the right of the panels show the position of the third ventricle, and the solid white lines encircle the SCN core.

\begin{tabular}{|lr}
\hline $\begin{array}{l}\text { http://jme.endocrinology-journals.org } \\
\text { https://doi.org/10.1530/JME-18-0062 }\end{array}$ & ( 2018 Society for Endocrinology \\
Published by Bioscientifica Ltd. \\
Printed in Great Britain
\end{tabular}


within the latest gene annotation containing 16052 annotated genes.

\section{cRNA array hybridization}

To further corroborate the immunohistochemical results and to demonstrate diurnal patterns of gene expression in the SCN, gene microarrays and qRT-PCR, were used to compare SCN gene expression profiles of old males (21-26 years) collected either during the daytime (1000$1100 \mathrm{~h}(\mathrm{ZT} 3-4, n=7))$ or $12 \mathrm{~h}$ later during the night (22:00-23:00h (ZT15-16, $n=5)$ ). During the nighttime tissue collections, the animals' eyes were covered to prevent potential negative impact of light exposure on mRNA expression in postmortem tissue experiments. The SCN RNA samples were extracted as described earlier and analyzed by the OHSU Gene Profiling Shared Resource. Briefly, target labeling was performed using total RNA samples that were prepared for array hybridization by synthesizing cDNA using the Ovation Pico WTA RNA Amplification System v.2 (NuGEN Technologies, San Carolos, CA, USA). Array hybridization and processing was performed by injecting the hybridization cocktail into a cartridge containing the GeneChip Rhesus Gene 1.0 ST array (Affymetrix, Thermo Fisher Scientific Inc.) containing 37,375 gene-level probe sets. The arrays were incubated, washed and stained on a GeneChip Fluidics Station 450 (Affymetrix, Thermo Fisher Scientific Inc.) using the Hybridization Wash and Stain kit (Affymetrix, Thermo Fisher Scientific Inc.). They were then scanned using a GeneChip Scanner 3000 7G with an autoloader (Affymetrix, Thermo Fisher Scientific Inc.) and analyzed using the Affymetrix Command Console (AGCC) v.3.1.1 software; the expression analysis was performed using Affymetrix Expression Console Software 1.4. Probe cell intensity files (CEL) for each of the experimental samples were uploaded into the Affymetrix Expression Console Software 1.4, and a multi-array analysis incorporating all the samples of the study group was performed using the Robust Multi-Array Average (RMA)-Sketch normalization and summarization algorithm for all exon transcripts (Noriega et al. 2010, Eghlidi et al. 2017).

\section{Quantitative RT-PCR}

To corroborate the gene array data from the diurnal study, selected mRNA expression patterns were also quantified by qRT-PCR, using a QuantStudio $12 \mathrm{~K}$ Flex thermocycler (Life Technologies). Primer (CLOCK, Forward-TCCATGCTTTCCTGGTAATGCT Reverse-CAAGAGCCTTTATGTTTTCGTAAAAA;
NPAS2, Forward-CCTCCTGTAACGGTTTTGACAAC Reverse-TGAAGCAAACCTCCTTTCCTAGTG and PER2, Forward-CGTGTGCAACGGAGCAGAT ReverseACTGACACGGCAAAAGAAAGATT) and probe (CLOCK, 6FAM-ACAAATCCACTGTTCTGCAG-MGB; NPAS2, 6FAM-CCCTTTCAAGACCTTG-MGB and PER2, 6FAMTTTTACTCAAGAATGCATGGAG-MGB) sequences were obtained using Primer Express Software and NCBI reference sequences (CLOCK, XM_015138689; PER2, XM_015111477 and NPAS2, XM_015112969). Initially, pooled cDNA was used to create standard curves for each gene, and the experimental samples were subsequently diluted to fall within the linear part of the curve. The PCR mixtures contained $5 \mu \mathrm{L}$ TaqMan Gene Expression Master Mix, $2.15 \mu \mathrm{L}$ water, $0.3 \mu \mathrm{L}$ each specific forward and reverse primer (300 nM final concentration), $0.25 \mu \mathrm{L}$ specific probe (250 nM final concentration) and $2 \mu \mathrm{L}$ cDNA. The realtime qPCR reaction sequence included $10-\mathrm{min}$ incubation at $95^{\circ} \mathrm{C}$, followed by 40 cycles of $15 \mathrm{~s}$ at $95^{\circ} \mathrm{C}, 1 \mathrm{~min}$ at $60^{\circ} \mathrm{C}, 1 \mathrm{~min}$ at $60^{\circ} \mathrm{C}$ and $15 \mathrm{~s}$ at $95^{\circ} \mathrm{C}$. Automatic baseline and threshold levels were determined by QuantStudio $12 \mathrm{~K}$ Flex thermocycler detection system software, and the final expression values were normalized to the arithmetic mean of ALG9 and RPL13A (Noriega et al. 2010, Eghlidi et al. 2010, 2015). For each gene, samples from all the animals were examined in triplicate using the same 384-well optical plate. A negative control included the omission of cDNA templates from the reaction mixture.

\section{Statistical analysis}

Comparisons between data means were made using unpaired Student's $t$-tests, and significance was considered at $P<0.05$. Where applicable, a false discovery rate (FDR) correction for multiple comparisons $P$ value was reported (Reiner et al. 2003).

\section{Results}

Spatial organization of immuno-positive neurons in the SCN of young and old rhesus macaques

Using immunohistochemical markers, the SCN was localized to a brain region that was ventral to the paraventricular nucleus, lateral to the third ventricle and just dorsal of the chiasma. The location of AVP immuno-positive neurons extended from the rostral to caudal shell of the SCN. Overall, the AVP immunoreactivity appeared less intense in the old animals than in the young animals, but some AVP neurons were still 
visible in the dorsal rostral medial shell of the SCN with fibers projecting from the medial caudal region of the SCN to the subparaventricular and paraventricular nuclei (Fig. 1A). In both young and old animals, VIP immuno-reactivity was evident in the core region of the SCN, with fibers projecting dorsally to the SCN shell and caudally to the sub-paraventricular zone (Fig. 1B). CALB immuno-reactivity was evident in the nucleus and cell bodies of many neurons (Fig. 1C). The core clock protein, REV-ERB $\alpha$, was expressed throughout the SCN, showing colocalization with AVP and VIP (Fig. 1D). Overall, there appeared to be fewer AVP, VIP and REV-ERB $\alpha$, but not CALB, neurons in the SCNs of the old animals, although the location and general anatomy of the SCN in the young and old animals was similar.

\section{Corroboration of SCN localization}

Based on the immunohistochemical coordinates of the SCN (Fig. 1), we isolated RNA form putative SCN regions of frozen hypothalamic blocks and used RNA-seq to compare gene expression intensities in the SCN samples to those in the amygdala and adrenal gland. Known to be unique to the SCN, AVP $(\mathrm{FPKM}=607.35)$ and $C A L B$ (FPKM $=42.54)$ mRNA were both highly expressed in the SCN of the animal obtained between 1000 and $1100 \mathrm{~h}$ (ZT3-4), as were GABA transporters SLC6A1 (FPKM = 42.33) and SLC6A11 (FPKM=55.67). As expected, numerous clock genes were also expressed in the SCN, including CRY2 (FPKM = 12.69), NR1D1 (FPKM=17.34) and CSNK1E (FPKM=25.64). VIP, PROK2, VIP1R, AVPR1A, AVPR1B, SLC6A3, SLC6A4, SLC17A6, SLC32A1, PER1, PER2, CLOCK, NPAS2, CRY1 and ARNTL showed a lower level of expression (FPKM < 10). Note that SLC17A7, VIPR2, PER3 and $D E C 1$ were not included on the aligner. For reference, the amygdala did not show enrichment of $A V P=2.94$, but expressed CALB $(\mathrm{FPKM}=22.52)$, SLC17A6 $(\mathrm{FPKM}=23.06)$ and SLC6A3 $(\mathrm{FPKM}=113.72)$, as well as expression of core circadian clock genes (PER1; FPKM $=10.74$, NPAS2 $=12.72, N R 1 D 1=19.82, C R Y 2=21.97)$. The adrenal gland was enriched for steroidogenic enzymes CYP17A1 $(\mathrm{FPKM}=6173.06)$ and HSD3B2 (FPKM $=2138.99)$ and core clock genes (NR1D1; FPKM $=12.59, C R Y 2=11.84$, PER1 $=24.08, A R N T L=13.08)$, but not $A V P(F P K M=0.46)$ or $C A L B(F P K M=1.05)$.

The RNA-seq results, combined with the immunohistochemical data, gave us confidence that we were accurately sub-dissecting the SCN. For further assurance, we used gene microarrays to compare the daytime vs nighttime gene expression in the putative
SCNs from old males. Although many genes showed diurnal differences in expression across the whole transcriptome, our analysis focused on genes integral to the function of the SCN (Fig. 2). As expected, several of these genes (i.e., NPAS2, NR1D1, SLC6A1 and SLC17A7) showed significant diurnal differences in expression $(P<0.05$, FDR correction for multiple comparisons). We subsequently examined diurnal gene expression differences using qRT-PCR by focusing on CLOCK, PER2 and NPAS2 (i.e., core clock genes that show a high level of expression in the SCN). Similar to the gene microarray results, NPAS2 was more highly expressed at 22:00-23:00 h compared to 10:00-11:00 h (Fig. 3). No other differences in clock-related gene expression were detected between these two time points.

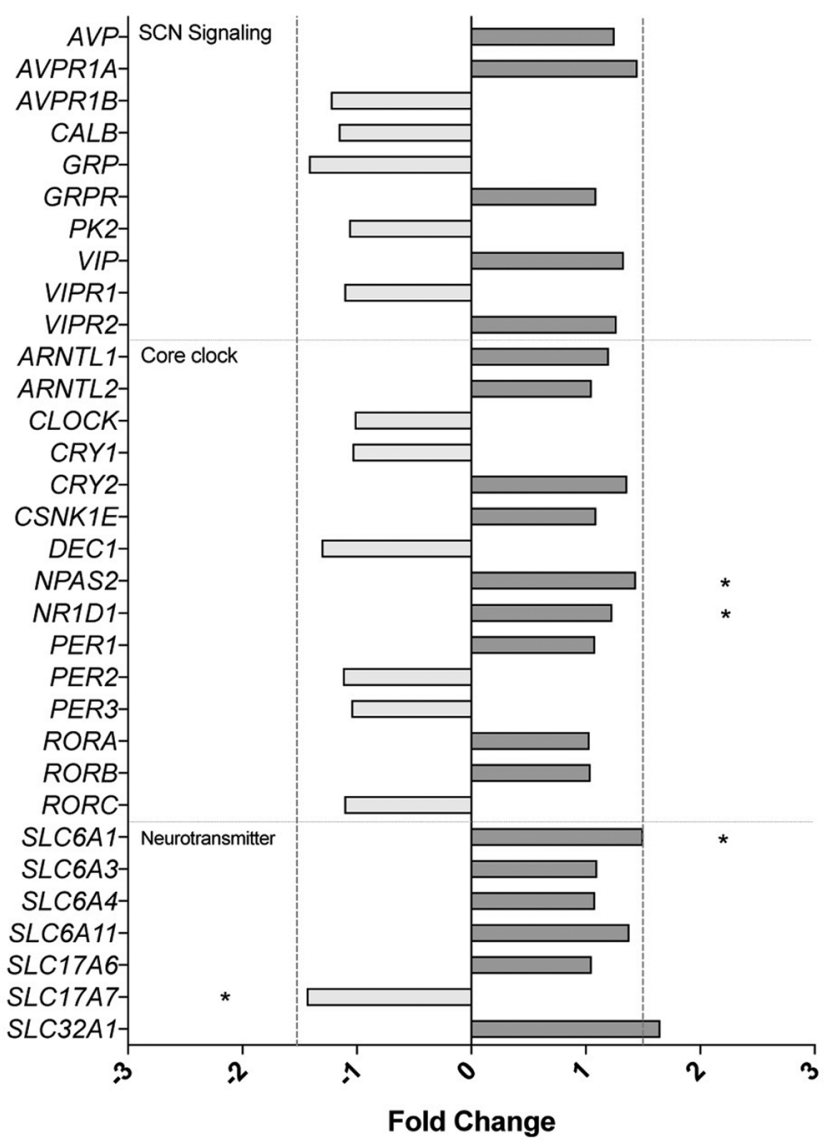

Figure 2

Diurnal gene expression profiling in the suprachiasmatic nucleus $(\mathrm{SCN})$ of old rhesus macaques using gene microarrays. NPAS2, NR1D1, SLC6A1 and SLC17A7 were statistically significant across the whole array $\left({ }^{*} P<0.05\right.$, post hoc false discovery rate). SLC32A1 had a $>1.5$-fold change, but this was not statistically significant. Higher nighttime expression (dark gray bars) vs higher daytime expression (light gray bars). 


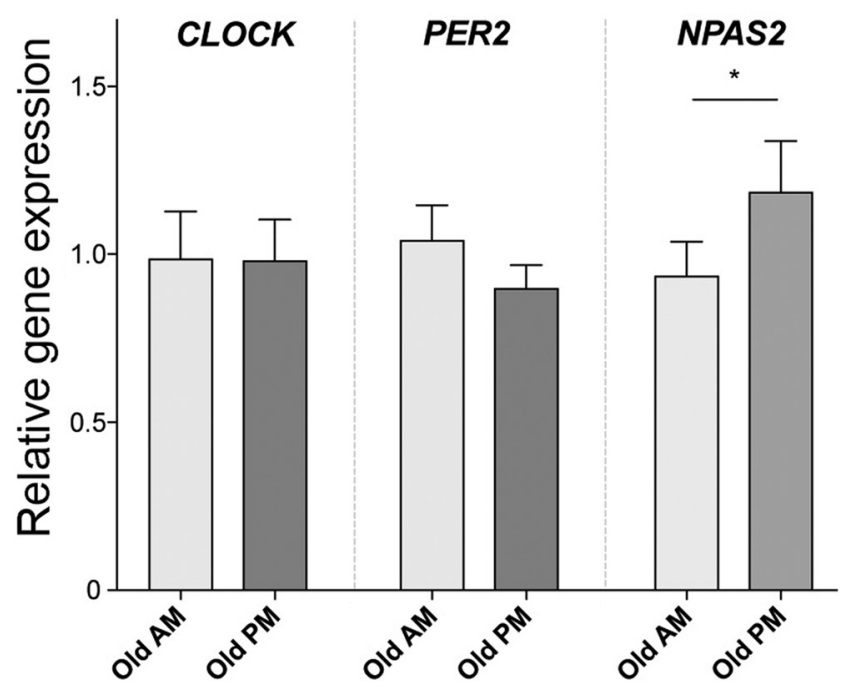

Figure 3

Diurnal gene expression profiling in the suprachiasmatic nucleus (SCN) of old rhesus macaques, using quantitative real-time PCR. The results are expressed relative to the arithmetic mean of a normalizer index (ALG9 and RPL13A). The values represent means ( \pm S.E.M.S) from the old males at 11 AM (light gray bars) and from old males at 11 PM (dark gray bars). The expression of NPAS2 was significantly higher at night relative to the daytime $\left({ }^{*} P<0.05\right)$. No other significant differences in gene expression were detected.

\section{Age-related gene expression changes within the SCN}

Using gene microarrays, we compared SCN-specific gene expression profiles of young and old rhesus males. Although VIP showed a large daytime age-related foldincrease, this was found to be statistically insignificant (Fig. 4). No other age-related changes in expression for any of the SCN-specific genes were detected. To corroborate some of the negative findings, we subsequently used qRT-PCR to compare the expression of CLOCK, PER2 and NPAS2, between the young and old animals. Similar to the gene microarray results, however, none of these genes showed an age-related change in the level of expression (Fig. 5).

\section{Discussion}

Aging is associated with changes in homeostatic function, such as disrupted hormone rhythms and wake-sleep cycles (Duffy et al. 2002, Yoon et al. 2003, Downs \& Urbanski 2006, Downs et al. 2007, Urbanski 2011). Perturbed circadian rhythms have been associated with disease, behavioral and cognitive deficits, as well as increased mortality (Davidson et al. 2006, Urbanski et al. 2011, Engelberth et al. 2013). Although the SCN plays

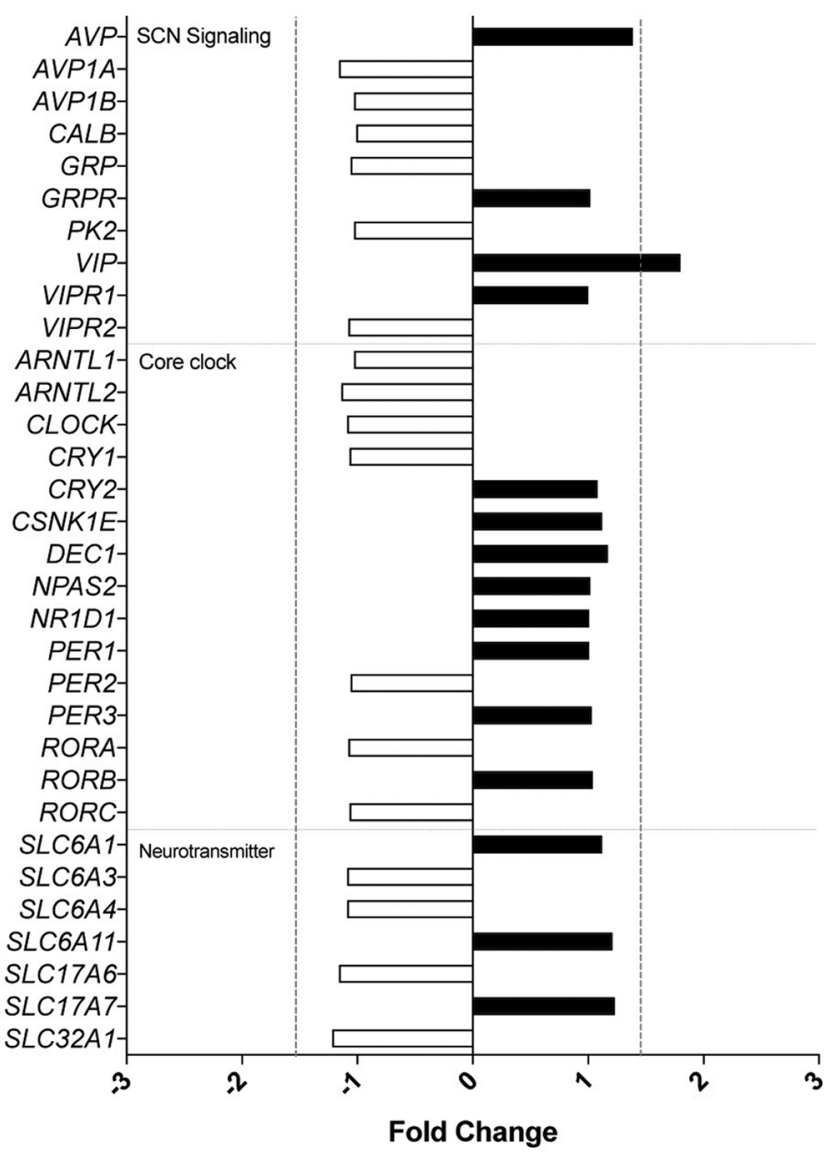

Figure 4

Gene expression profiling in the suprachiasmatic nucleus (SCN) of young adult and old rhesus macaques, using gene arrays. Fold-change higher gene expression in aged animals is shown by black bars. There was no obvious difference in gene expression between the young and old animals; although VIP showed a $>1.5$-fold increase in expression between young and old animals, this was not statistically significant.

a primary role in maintaining circadian rhythms, the SCN of primates has not been characterized extensively (Moore 1993). Therefore, our preliminary objective was to characterize the organization of the rhesus macaque SCN. As expected based on rodent studies, we found that neuropeptidergic cells of the rhesus macaque SCN exist in heterogeneous populations. CALB neurons were found clustered in a similar subnucleus as described in the hamster and the mouse lemur (Jobst et al. 2004, Cayetanot et al. 2007). In contrast, VIP neurons formed a distinctive core population in the rostral portion of the SCN, encapsulated by a shell of AVP neurons, which extended up toward the paraventricular nucleus. Together, these immunohistochemical findings are in general agreement with observations previously reported by Moore (1993) and emphasize that the overall anatomy of the rhesus macaque SCN is well preserved even into old age. 


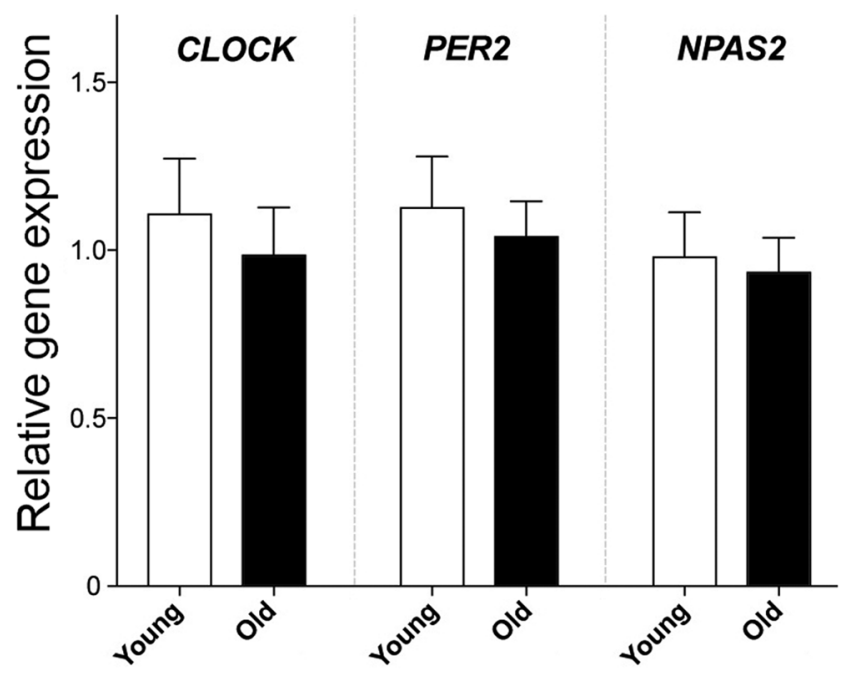

Figure 5

Gene expression profiling in the suprachiasmatic nucleus (SCN) of young adult and old rhesus macaques, using quantitative real-time PCR. The results are expressed relative to the arithmetic mean of a normalizer index (ALG9 and RPL13A). The values represent means ( \pm S.E.M.S) from the young (white bars) and old (black bars) males. No significant age-related differences in gene expression were detected.

Our main objective, however, was to examine age-related gene expression changes in the SCN that may contribute to the etiology of age-related disorders involving disrupted circadian rhythms. Therefore, prior to necropsy, we used actigraphy to verify that the old males from the present study indeed had significantly attenuated daytime activity levels compared to the young adults. As previously reported (Urbanski 2017), the mean \pm S.E.M. daytime activity level of the old animals $(147.6 \pm 24.2)$ was significantly $(P<0.01)$ lower than that of the young animals $(215.3 \pm 15.3)$. Therefore, we expected to find significant age-related differences in the expression of many SCN genes that play a key role in circadian timekeeping. However, we found none. There are a few possible reasons for this negative finding. Firstly, analysis of gene expression using microarrays has some inherent limitations. Although we used a very conservative 1.0-foldchange cut off in our analysis, we filtered out genes that did not show a robust $(P<0.05)$ effect. Therefore, we may have missed genes that showed subtle statistically insignificant but physiologically important age-related changes in expression. However, using qRT-PCR, we similarly failed to detect a significant age-related change in the expression of key core clock genes within the SCN. The general agreement between the gene microarray and the qRT-PCR results, despite the difference in sequences targeted by the two methodologies, helps to validate the negative data. Although significant age-related gene expression changes have previously been observed in the hypothalamic arcuate nucleus of women and female rhesus macaques (Rance \& Young 1991, Eghlidi et al. 2010, Eghlidi \& Urbanski 2015), similar changes were not observed in males (Eghlidi et al. 2017), suggesting that the more profound age-related decrease in circulating sex-steroid concentrations in females may have played a causal role. Similarly, because the SCN of monkeys and humans expresses estrogen receptors (Gundlah et al. 2000, Kruijver \& Swaab 2002) and shows some sex differences (Bailey \& Silver 2014), it is plausible that more obvious age-related gene expression changes may be found in the $\mathrm{SCN}$ of females than males.

Secondly, it is well established that many genes have a diurnal pattern of expression (Lemos et al. 2006, Sitzmann et al. 2010, Zhang et al. 2014), and so it is possible that age-related changes in expression level might only be obvious during the peak of their diurnal expression and not at other times of the day. For example, NPAS2 was more highly expressed in the SCN during the night than during the daytime, which is in agreement with our previous observation in the rhesus macaque adrenal gland (Lemos et al. 2006). Therefore, it is unclear if age-related changes in the expression of NPAS2 and some other genes would have been more obvious had we collected SCN samples from the young and old animals during the night, rather than during the daytime. On the other hand, this explanation would not account for the lack of age-related changes in PER2 and CLOCK expression, which appeared to show peak expression during the daytime (i.e., time of day when the SCN samples were collected for the aging study). To completely rule out a potential masking effect of 'time of day' on age-related gene expression changes would have required collecting SCN tissues from many more young and old animals at multiple time points across the 24-h day, something that is not feasible given the high cost and scarcity of postmortem primate tissue, especially from old rhesus macaques. Interestingly, in a recent study of circadian gene expression in baboons (Mure et al. 2018), rhythmically expressed genes in the SCN showed a very narrow peak at ZT2. Therefore, collection of tissues at ZT3-4 in the present study represents a pragmatic time point for studying age-related gene expression changes in a diurnal nonhuman primate.

Thirdly, microarray and qRT-PCR analysis is confined to the RNA level, yet, the circadian core clock mechanisms itself comprise both transcriptional and translational components. Therefore, we cannot exclude the possibility that significant age-related changes in the SCN occur at the protein level. Although we performed 
immunohistochemistry for AVP, VIP, CALB and REV$\mathrm{ERB} \alpha$ (i.e., proteins that play important roles in the circadian organization of the $\mathrm{SCN}$ ), this non-quantitative methodology does not allow us to make any definitive statements about changes in protein expression during aging. Overall, however, the old animals appeared to show fewer cells in the SCN that were immunopositive for AVP, VIP and REV-ERB $\alpha$, but not CALB (data not shown). These observations are in general agreement with previous reports of an age-related decrease of AVP and VIP protein expression in the SCN of the mouse lemur (Cayetanot et al. 2005, Aujard et al. 2006), and with some reports of decreased AVP and VIP immunostaining in the SCN of elderly humans (Hofman \& Swaab 1994, Hofman et al. 1996, Harper et al. 2008).

Despite some limitations, results from the present gene microarray and qRT-PCR studies strongly suggest that the primate SCN is relatively resistant to aging, at least at the mRNA level and at least in males. The results also demonstrate that the SCN of old rhesus macaques shows normal spatial organization of neuropeptides that are important for normal circadian function (i.e., AVP, VIP, CALB and REV-ERB $\alpha$ ) and maintains diurnal gene expression patterns for several other genes including NPAS2 and NR1D1, both of which were more highly expressed at night. The microarray analysis also gave us an opportunity to examine the diurnal expression rhythms of a number of transporter genes in the SCN of the old animals. Although the SCN is known to express GABA transporters (1 and 3), and Vglut1, the expression profiles of these transporter genes had not previously been studied in primates (Ziegler et al. 2002, Moldavan et al. 2015). Our results show that SLC6A1 (VGAT1) and SLC17A7 (VGLUT1) still maintained a diurnal expression pattern in the SCN of old animals, but interestingly, the two transporters showed different times of peak expression, with $S L C 6 A 1$ being more highly expressed at night and SLC17A7 being more highly expressed during the day. Diurnal expression of these transporters suggests that classic neurotransmitter signaling within the SCN is likely to be maintained even in aged animals.

Importantly, from a physiological perspective, there does not appear to be any significant difference in daytime gene expression between the young and old males, despite the latter showing significantly attenuated daytime activity levels (Urbanski 2017). This age-related stability within the transcriptional components of the master circadian clock in the SCN suggests that age-related attenuation of human circadian activity-rest cycles may instead reside at the translational or electrophysiological level within the SCN or stem from disruption of other components of the circadian regulating system, such as desynchronization of subordinate oscillators in other parts of the body.

Declaration of interest

The authors declare that there is no conflict of interest that could be perceived as prejudicing the impartiality of the research reported.

\section{Funding}

This work was supported by National Institutes of Health grants: AG029612, AG-036670, HL-007901, OD-010426, OD-011092 and OD-011895.

\section{Author contributions statement}

D H E performed experiments, analyzed the data and prepared the manuscript. V T G helped with the data analysis. S L L and D I B helped with the immunohistochemistry. H F U and S G K helped with the design of the study, collected tissues and helped with preparation of the manuscript.

\section{Acknowledgements}

The authors would like to thank the ONPRC Division of Comparative Medicine for assistance with caring for the animals. They would also like to thank the ONPRC Molecular and Cell Biology Core and the ONPRC Imaging and Morphology Core for assistance with mRNA quantitation and confocal microscopy, and the OHSU Gene Profiling Shared Resource for gene expression profiling using Affymetrix Gene Chip arrays.

\section{References}

Abrahamson EE \& Moore RY 2001 Suprachiasmatic nucleus in the mouse: retinal innervation, intrinsic organization and efferent projections. Brain Research 916 172-191. (https://doi.org/10.1016/ S0006-8993(01)02890-6)

An S, Tsai C, Ronecker J, Bayly A \& Herzog ED 2012 Spatiotemporal distribution of vasoactive intestinal polypeptide receptor 2 in mouse suprachiasmatic nucleus. Journal of Comparative Neurology $\mathbf{5 2 0}$ 2730-2741. (https://doi.org/10.1002/cne.23078)

An S, Harang R, Meeker K, Granados-Fuentes D, Tsai CA, Mazuski C, Kim J, Doyle FJ 3rd, Petzold LR \& Herzog ED 2013 A neuropeptide speeds circadian entrainment by reducing intercellular synchrony. PNAS 110 E4355-E4361. (https://doi.org/10.1073/pnas.1307088110)

Anders S \& Huber W 2010 Differential expression analysis for sequence count data. Genome Biology 11 R106. (https://doi.org/10.1186/ gb-2010-11-10-r106)

Aton SJ, Huettner JE, Straume M \& Herzog ED 2006 GABA and Gi/o differentially control circadian rhythms and synchrony in clock neurons. PNAS 103 19188-19193. (https://doi.org/10.1073/ pnas.0607466103)

Aujard F, Cayetanot F, Bentivoglio M \& Perret M 2006 Age-related effects on the biological clock and its behavioral output in a primate. Chronobiology International 23 451-460. (https://doi. org/10.1080/07420520500482090)

Bailey M \& Silver R 2014 Sex differences in circadian timing systems: implications for disease. Frontiers in Neuroendocrinology 35 111-139. (https://doi.org/10.1016/j.yfrne.2013.11.003)

Burton KJ, Li X, Li B, Cheng M, Urbanski, HF \& Zhou QY 2016 Expression of prokineticin 2 and its receptor in the macaque 
monkey brain. Chronobiology International 33 191-199. (https://doi. org/10.3109/07420528.2015.1125361)

Cayetanot F, Bentivoglio M \& Aujard F 2005 Arginine-vasopressin and vasointestinal polypeptide rhythms in the suprachiasmatic nucleus of the mouse lemur reveal aging-related alterations of circadian pacemaker neurons in a non-human primate. European Journal of Neuroscience 22 902-910. (https://doi.

org/10.1111/j.1460-9568.2005.04268.x)

Cayetanot F, Deprez J \& Aujard F 2007 Calbindin D28K protein cells in a primate suprachiasmatic nucleus: localization, daily rhythm and age-related changes. European Journal of Neuroscience 26 2025-2032. (https://doi.org/10.1111/j.1460-9568.2007.05826.x)

Chee CA, Roozendaal B, Swaab DF, Goudsmit E \& Mirmiran M 1988 Vasoactive intestinal polypeptide neuron changes in the senile rat suprachiasmatic nucleus. Neurobiology of Aging 9 307-312. (https:// doi.org/10.1016/S0197-4580(88)80070-8)

Davidson AJ, Sellix MT, Daniel J, Yamazaki S, Menaker M \& Block GD 2006 Chronic jet-lag increases mortality in aged mice. Current Biology 16 R914-R916. (https://doi.org/10.1016/j.cub.2006.09.058)

Downs JL \& Urbanski HF 2006 Neuroendocrine changes in the aging reproductive axis of female rhesus macaques (Macaca mulatta). Biology of Reproduction 75 539-546. (https://doi.org/10.1095/ biolreprod.106.051839)

Downs JL, Dunn MR, Borok E, Shanabrough M, Horvath TL, Kohama SG \& Urbanski HF 2007 Orexin neuronal changes in the locus coeruleus of the aging rhesus macaque. Neurobiology of Aging 28 1286-1295. (https://doi.org/10.1016/j.neurobiolaging.2006.05.025)

Duffy JF, Zeitzer JM, Rimmer DW, Klerman EB, Dijk DJ \& Czeisler CA 2002 Peak of circadian melatonin rhythm occurs later within the sleep of older subjects. American Journal of Physiology: Endocrinology and Metabolism 282 E297-E303.

Eghlidi DH \& Urbanski HF 2015 Effect of age and estradiol on gene expression in the rhesus macaque hypothalamus. Neuroendocrinology 101 236-245. (https://doi.org/10.1159/000381063)

Eghlidi DH, Haley GE, Noriega NC, Kohama SG \& Urbanski HF 2010 Influence of age and 17beta-estradiol on kisspeptin, neurokinin B, and prodynorphin gene expression in the arcuate-median eminence of female rhesus macaques. Endocrinology 151 3783-3794. (https:// doi.org/10.1210/en.2010-0198)

Eghlidi DH, Garyfallou VT, Kohama SG \& Urbanski HF 2017 Ageassociated gene expression changes in the arcuate nucleus of male rhesus macaques. Journal of Molecular Endocrinology 59 141-149. (https://doi.org/10.1530/JME-17-0094)

Engelberth RCGJ, Pontes ALB, Fiuza FP, Silva KDA, Resende NS, Azevedo CVM, Costa MSMO, Cavalcante JC, Nascimento ES Jr, Gavioli EC, et al. 2013 Changes in the suprachiasmatic nucleus during aging: implications for biological rhythms. Psychology and Neuroscience 6 287-297. (https://doi.org/10.3922/j.psns.2013.3.07)

Engelberth RC, Silva KD, Azevedo CV, Gavioli EC, dos Santos JR, Soares JG, Nascimento Junior ES, Cavalcante JC, Costa MS \& Cavalcante JS 2014 Morphological changes in the suprachiasmatic nucleus of aging female marmosets (Callithrix jacchus). Biomed Research International 2014 243825. (https://doi. org/10.1155/2014/243825)

Farajnia S, Michel S, Deboer T, vanderLeest HT, Houben T, Rohling JH, Ramkisoensing A, Yasenkov R \& Meijer JH 2012 Evidence for neuronal desynchrony in the aged suprachiasmatic nucleus clock. Journal of Neuroscience 32 5891-5899. (https://doi.org/10.1523/ JNEUROSCI.0469-12.2012)

Gao B, Fritschy JM \& Moore RY 1995 GABAA-receptor subunit composition in the circadian timing system. Brain Research 700 142-156. (https://doi.org/10.1016/0006-8993(95)00944-L)

Gundlah C, Kohama SG, Mirkes SJ, Garyfallou VT, Urbanski HF \& Bethea CL 2000 Distribution of estrogen receptor beta (ERß) mRNA in the macaque brain and its regulation by ovarian steroids.
Molecular Brain Research 76 191-204. (https://doi.org/10.1016/S00068993(99)02475-0)

Haley GE, Eghlidi DH, Kohama SG, Urbanski HF \& Raber J 2012 Association of microtubule associated protein-2, synaptophysin, and apolipoprotein E mRNA and protein levels with cognition and anxiety levels in aged female rhesus macaques. Behavioural Brain Research 232 1-6. (https://doi.org/10.1016/j.bbr.2012.03.032)

Hamada T, LeSauter J, Lokshin M, Romero MT, Yan L, Venuti JM \& Silver R 2003 Calbindin influences response to photic input in suprachiasmatic nucleus. Journal of Neuroscience 23 8820-8826. (https://doi.org/10.1523/JNEUROSCI.23-26-08820.2003)

Harper DG, Stopa G, Kuo-Leblanc V, McKee AC, Asayama K, Volicer L, Kowall N \& Satlin A 2008 Dorsomedial SCN neuronal subpopulations subserve different functions in human dementia. Brain 131 1609-1617. (https://doi.org/10.1093/brain/awn049)

Hastings MH, Reddy AB \& Maywood ES 2003 A clockwork web: circadian timing in brain and periphery, in health and disease. Nature Reviews: Neuroscience 4 649-661. (https://doi.org/10.1038/ nrn1177)

Hofman MA \& Swaab DF 1994 Alterations in circadian rhythmicity of the vasopressin-producing neurons of the human suprachiasmatic nucleus (SCN) with aging. Brain Research 651 134-142. (https://doi. org/10.1016/0006-8993(94)90689-0)

Hofman MA, Zhou JN \& Swaab DF 1996 Suprachiasmatic nucleus of the human brain: an immunocytochemical and morphometric analysis. Anatomical Record 244 552-562. (https://doi.org/10.1002/(SICI)10970185(199604)244:4<552::AID-AR13>3.0.CO;2-O)

Irwin RP \& Allen CN 2009 GABAergic signaling induces divergent neuronal $\mathrm{Ca} 2+$ responses in the suprachiasmatic nucleus network. European Journal of Neuroscience 30 1462-1475. (https://doi. org/10.1111/j.1460-9568.2009.06944.x)

Jiao Y, Sun Z, Lee T, Fusco FR, Kimble TD, Meade CA, Cuthberton S \& Reiner A 1999 A simple and sensitive antigen retrieval method for free-floating and slide-mounted tissue sections. Journal of Neuroscience Methods 93 149-162. (https://doi.org/10.1016/S0165-0270(99)00142-9)

Jobst EE, Robinson DW \& Allen CN 2004 Potential pathways for intercellular communication within the calbindin subnucleus of the hamster suprachiasmatic nucleus. Neuroscience 123 87-99. (https:// doi.org/10.1016/j.neuroscience.2003.08.059)

Kawakami F, Okamura H, Tamada Y, Maebayash, Y, Fukui K \& Ibata Y 1997 Loss of day-night differences in VIP mRNA levels in the suprachiasmatic nucleus of aged rats. Neuroscience Letters 222 99-102. (https://doi.org/10.1016/S0304-3940(97)13355-9)

Kolker DE, Fukuyama H, Huang DS, Takahashi JS, Horton TH \& Turek FW 2003 Aging alters circadian and light-induced expression of clock genes in golden hamsters. Journal of Biological Rhythms $\mathbf{1 8}$ 159-169. (https://doi.org/10.1177/0748730403251802)

Kruijver FPM \& Swaab DF 2002 Sex hormone receptors are present in the human suprachiasmatic nucleus. Neuroendocrinology 75 296-305. (https://doi.org/10.1159/000057339)

Lemos DR, Downs JL \& Urbanski HF 2006 Twenty-four-hour rhythmic gene expression in the rhesus macaque adrenal gland. Molecular Endocrinology 20 1164-1176. (https://doi.org/10.1210/me.2005-0361)

Lohse M, Bolger AM, Nagel A, Fernie AR, Lunn JE, Stitt M \& Usadel B 2012 RobiNA: a user-friendly, integrated software solution for RNASeq-based transcriptomics. Nucleic Acids Research 40 W622-W627. (https://doi.org/10.1093/nar/gks540)

Maywood ES, Reddy AB, Wong GK, O'Neill JS, O'Brien JA, McMahon DG, Harmar AJ, Okamura H \& Hastings MH 2006 Synchronization and maintenance of timekeeping in suprachiasmatic circadian clock cells by neuropeptidergic signaling. Current Biology 16 599-605. (https://doi.org/10.1016/j. cub.2006.02.023)

Maywood ES, O'Neill JS, Chesham JE \& Hastings MH 2007 Minireview: the circadian clockwork of the suprachiasmatic nuclei - analysis of a
(C) 2018 Society for Endocrinology Published by Bioscientifica Ltd. Printed in Great Britain 
cellular oscillator that drives endocrine rhythms. Endocrinology 148 5624-5634. (https://doi.org/10.1210/en.2007-0660)

Moldavan M, Cravetchi O, Williams M, Irwin RP, Aicher SA \& Allen CN 2015 Localization and expression of GABA transporters in the suprachiasmatic nucleus. European Journal of Neuroscience $\mathbf{4 2}$ 3018-3032. (https://doi.org/10.1111/ejn.13083)

Moore RY 1993 Organization of the primate circadian system. Journal of Biological Rhythms 8 S3-S9.

Mure LS, Le HD, Benegiamo G, Chang MW, Rios L, Jillani N, Ngotho M, Kariuki T, Dkhissi-Benyahya O, Cooper HM, et al. 2018 Diurnal transcriptome atlas of a primate across major neural and peripheral tissues. Science 359. (https://doi.org/10.1126/science.aao0318).

Nakamura TJ, Nakamura W, Yamazaki S, Kudo T, Cutler T, Colwell CS \& Block GD 2011 Age-related decline in circadian output. Journal of Neuroscience 31 10201-10205. (https://doi.org/10.1523/ JNEUROSCI.0451-11.2011)

Noriega NC, Eghlidi DH, Garyfallou VT, Kohama SG, Kryger SG \& Urbanski HF 2010 Influence of 17beta-estradiol and progesterone on GABAergic gene expression in the arcuate nucleus, amygdala and hippocampus of the rhesus macaque. Brain Research 1307 28-42. (https://doi.org/10.1016/j.brainres.2009.10.011)

Rance NE \& Young WS 3rd 1991 Hypertrophy and increased gene expression of neurons containing neurokinin-B and substance-P messenger ribonucleic acids in the hypothalami of postmenopausal women. Endocrinology 128 2239-2247. (https://doi.org/10.1210/ endo-128-5-2239)

Reiner A, Yekutieli D \& Benjamini Y 2003 Identifying differentially expressed genes using false discovery rate controlling procedures. Bioinformatics 19 368-375. (https://doi.org/10.1093/bioinformatics/ btf877)

Roberts DE, Killiany RJ \& Rosen DL 2012 Neuron numbers in the hypothalamus of the normal aging rhesus monkey: stability across the adult lifespan and between the sexes. Journal of Comparative Neurology 520 1181-1197. (https://doi.org/10.1002/cne.22761)

Roozendaal B, van Gool WA, Swaab DF, Hoogendijk JE \& Mirmiran M 1987 Changes in vasopressin cells of the rat suprachiasmatic nucleus with aging. Brain Research 409 259-264. (https://doi. org/10.1016/0006-8993(87)90710-4)

Saper CB 2013 The central circadian timing system. Current Opinions in Neurobiology 23 747-751. (https://doi.org/10.1016/j.conb.2013.04.004)

Sartin JL \& Lamperti AA 1985 Neuron numbers in hypothalamic nuclei of young, middle-aged and aged male rats. Experientia 41 109-111. (https://doi.org/10.1007/BF02005902)

Sitzmann BD, Lemos DR, Ottinger MA \& Urbanski HF 2010 Effects of age on clock gene expression in the rhesus macaque pituitary gland. Neurobiology of Aging 31 696-705. (https://doi.org/10.1016/j. neurobiolaging.2008.05.024)

Urbanski HF 2011 Role of circadian neuroendocrine rhythms in the control of behavior and physiology. Neuroendocrinology 93 211-222. (https://doi.org/10.1159/000327399)

Urbanski HF 2017 Effect of androgen supplementation on 24-hour activityrest patterns of aged male rhesus macaques. Neurobiology of Aging $\mathbf{5 4}$ 100-102. (https://doi.org/10.1016/j.neurobiolaging.2017.02.020)

Weinert D 2000 Age-dependent changes of the circadian system. Chronobiology International 17 261-283. (https://doi.org/10.1081/CBI100101048)

Weinert H, Weinert D, Schurov I, Maywood ES \& Hastings MH 2001 Impaired expression of the mPer2 circadian clock gene in the suprachiasmatic nuclei of aging mice. Chronobiology International 18 559-565. (https://doi.org/10.1081/CBI-100103976)

Yoon IY, Kripke DF, Elliott JA, Youngstedt SD, Rex KM \& Hauger RL 2003 Age-related changes of circadian rhythms and sleep-wake cycles. Journal of the American Geriatrics Society 51 1085-1091. (https://doi.org/10.1046/j.1532-5415.2003.51356.x)

Ziegler DR, Cullinan WE \& Herman JP 2002 Distribution of vesicular glutamate transporter mRNA in rat hypothalamus. Journal of Comparative Neurology 448 217-229. (https://doi.org/10.1002/ cne.10257)

Zhang R, Lahens NL, Balance HI, Hughes ME \& Hogenesch JB 2014 A circadian gene expression atlas in mammals: implications for biology and medicine. PNAS 45 16219-16224. (https://doi.org/10.1073/ pnas.1408886111)

Received in final form 4 May 2018

Accepted 9 May 2018

Accepted Preprint published online 9 May 2018
(C) 2018 Society for Endocrinology Published by Bioscientifica Ltd. Printed in Great Britain 